\title{
7 - What type of Automated External Defibrillators (AEDs) do primary care Doctors want?
}

\author{
Authors; Dr. John Dowling, General Practitioner, North West Immediate Care \\ Programme. Ms. Mairead McFadden, Resuscitation Officer, HSE. Mr. Martin Quinn, \\ OHCAR Manager, NUI Galway.
}

Purpose of the study

The purpose of this study was to ascertain what type of AEDs a group of 33 General Practitioners (GPs), who had previously been supplied and used AEDs, would wish for as replacements for existing defibrillators. Previous studies have identified AEDs as the most suitable defibrillators for $\mathrm{GPs}^{1-3}$. This study aims to focus on the type of AEDs GPs preferred in advance of deciding to replace 108 AEDs to GPs in the North West region of Ireland.

\section{Materials and methods}

The survey was conducted among GPs attending for a teaching update on basic life support (BLS). The question posed was whether they considered it important to have a screen on an AED to allow monitoring of rhythms or would they be happy to have a model that simply delivered a shock. The replies received are as outlined in figure 1 below. Additional information obtained was that the majority of GPs stored their AEDs in their surgeries and considered it useful to have an AED located outside their surgery.

\section{Conclusion}

The study identified that $48 \%$ of GPs felt the presence of a monitor screen was useful while $26 \%$ felt it was not useful or served as a distraction. Analysis of cost of adding a monitor screen across three leading brands of AEDs was an extra $46 \%$ in cost. In the context of resupplying 108 GPs with AEDs as a public health initiative, the presence of a monitor screen would add significant additional cost for little quantifiable benefit.

\section{References}

1. Colquhoun M. Resuscitation by primary care doctors. Resuscitation (2006) 70,229-237.

2. Masterson S, Wright $P$, Dowling J, Swann D, Bury G, Murphy

A. Out-of-hospital cardiac arrest (OHCA) survival in rural Northwest Ireland: 17 years' experience. Emergency Medical Journal 2011; 28:437-438.

3. Bury G, Headon M, Egan M, Dowling J. Cardiac Arrest management in general practice in Ireland: a 5-year crosssectional study. BMJ Open 2013;3:e002563.

Doi:10.1136/bmjopen-2013-002563.

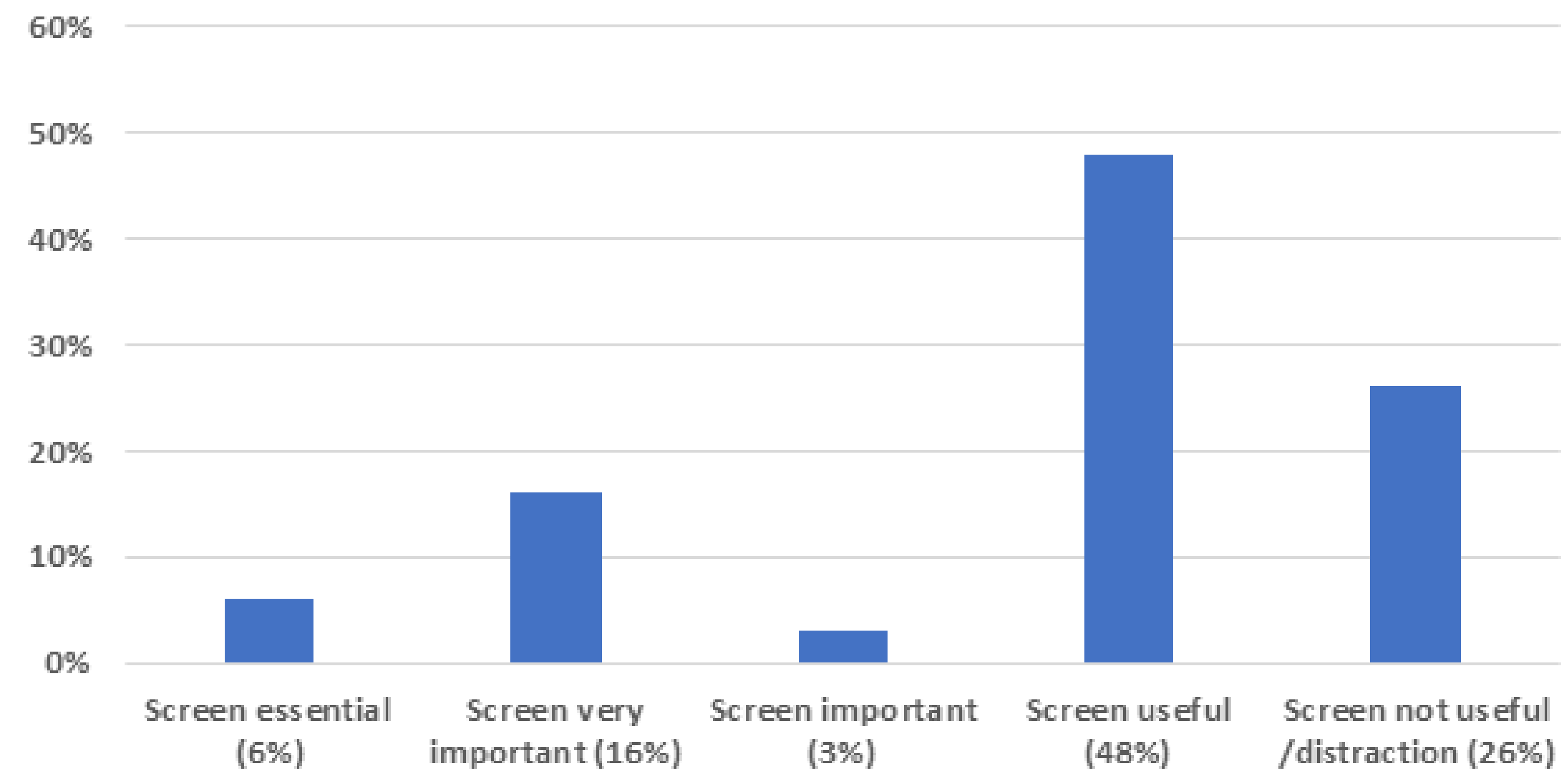

- Figure 1- Screen usefull ness ( $\mathrm{n}=33$ ) 